\section{OPEN ACCESS}

Edited by:

Ruth Grene,

Virginia Tech, USA

Reviewed by:

Vivekanand Tiwari,

Weizmann Institute of Science, Israe

Shaojun Xie,

Purdue University, USA

${ }^{*}$ Correspondence:

Chuanzhi Zhao

zhaochuanzhi@gmail.com

Specialty section: This article was submitted to

Plant Abiotic Stress,

a section of the journal

Frontiers in Plant Science

Received: 05 January 2017 Accepted: 18 April 2017

Published: 08 May 2017

Citation:

Wang J, Zhang Q, Cui F, Hou L, Zhao S, Xia H, Qiu J, Li T, Zhang Y,

Wang $X$ and Zhao C (2017)

Genome-Wide Analysis of Gene Expression Provides New Insights into

Cold Responses in Thellungiella

salsuginea. Front. Plant Sci. 8:713.

doi: 10.3389/fpls.2017.00713

\title{
Genome-Wide Analysis of Gene Expression Provides New Insights into Cold Responses in Thellungiella salsuginea
}

\begin{abstract}
Jiangshan Wang1,2, Quan Zhang ${ }^{3}$, Feng Cui', Lei Hou' ${ }^{1}$, Shuzhen Zhao', Han Xia', Jingjing Qiu',3, Tingting Li ${ }^{1}$, Ye Zhang ${ }^{1}$, Xingjun Wang ${ }^{1,3}$ and Chuanzhi Zhao ${ }^{1,3 *}$
\end{abstract}

'Biotechnology Research Center, Shandong Academy of Agricultural Sciences, Shandong Provincial Key Laboratory of Crop Genetic Improvement, Ecology and Physiology, Jinan, China, ${ }^{2}$ Yantai Institute of China Agricultural University, Yantai, China, ${ }^{3}$ College of Life Sciences, Shandong Normal University, Jinan, China

Low temperature is one of the major environmental stresses that affects plant growth and development, and leads to decrease in crop yield and quality. Thellungiella salsuginea (salt cress) exhibits high tolerance to chilling, is an appropriate model to investigate the molecular mechanisms of cold tolerance. Here, we compared transcription changes in the roots and leaves of $T$. salsuginea under cold stress using RNA-seq. We identified 2,782 and 1,430 differentially expressed genes (DEGs) in leaves and roots upon cold treatment, respectively. The expression levels of some genes were validated by quantitative real-time-PCR (qRT-PCR). Among these DEGs, 159 (11.1\%) genes in roots and 232 (8.3\%) genes in leaves were annotated as various types of transcription factors. We found that five aquaporin genes (three TIPS, one PIPS, and one NIPS) responded to cold treatment. In addition, the expression of COR47, ICE1, and CBF1 genes of DREB1/CBF-dependent cold signaling pathway genes altered in response to low temperature. KEGG pathway analysis indicated that these cold regulated genes were enriched in metabolism, photosynthesis, circadian rhythm, and transcriptional regulation. Our findings provided a complete picture of the regulatory network of cold stress response in T. salsuginea. These cold-responsive genes could be targeted for detail functional study and utilization in crop cold tolerance improvement.

Keywords: Thellungiella salsuginea, salt cress, cold stress, gene expression

\section{INTRODUCTION}

Plants generally are rooted in one place, and have to face drought, salinity, high temperature, cold, and other adverse stresses which may cause significant loss of crop yield (Boyer, 1982; Kawasaki and Bohnert, 2001). Low temperature is one of the major environmental stresses that affect plant growth and development, crop yield and quality. In plant tissues, the intercellular fluid generally has a higher freezing point than the intracellular fluid. When temperature decreased below freezing point, intercellular spaces of plant tissues form ice prior to intracellular region. So, the water potential decreases rapidly outside the cells, and causes the movement of water from inside the cell to the intercellular spaces. Consequently, cold stress could lead to severe cellular dehydration 
(Thomashow, 1998). In addition, low temperature can lead to the formation of adhesion between the intercellular ice and the cell walls and membranes (Levitt, 1980). Low temperature damage could lead to growth inhibition, wilting, and weak seedling.

During the evolution history, most plants developed the capacity to tolerate cold. Cold acclimation is a strategy for the plants to acquire freezing tolerance by a prior exposure to low nonfreezing temperature (Guy, 1990). To adapt to low temperature environment, many physiological and molecular changes occur during cold acclimation (Thomashow, 1999). Exposure to low temperature, linolenic acid and membrane lipid unsaturation increased, and the plasma membrane $\mathrm{H}^{+}$-ATPase activity increased and these changes are essential for the plants to withstand low temperature (Shi et al., 2008). In addition, calcium-dependent protein kinase confers cold tolerance via the regulation of calcium channel in plasma membrane (Xiong et al., 2002; Komatsu et al., 2007). Early studies identified a number of genes in plants which were response to cold treatment, and these genes were known as cold regulated (COR) genes (Thomashow, 1999; Lee et al., 2005). For example, a total of 939 cold regulated genes were identified in Arabidopsis thaliana (Lee et al., 2005). The cold regulated genes were involved in a variety of functions such as metabolism, protein synthesis, signal transduction, transcription regulation, and hormone biosynthesis and signaling (Thomashow, 1999; Lee et al., 2005).

Among these COR genes, a family of transcription factor known as C-repeat/dehydration-responsive element-binding $(C B F)$ was identified as the key factor to regulate response to cold stress in many plants (Gao et al., 2002; Xiong and Fei, 2006; Nakamura et al., 2011; Wisniewski et al., 2011). In Arabidopsis, three members of $C B F$ s were identified, including $C B F 1, C B F 2$, and CBF3 (also name as DREB1b, DREB1c, and DREB1a, respectively). Overexpression of $C B F 1$ induced $C O R$ genes and increased freeze tolerance of the transgenic plants (Jaglo-Ottosen et al., 1998; Yamaguchi-Shinozaki and Shinozaki, 2001). Deletion of all three $C B F$ genes the transgenic plants are extremely sensitive to freezing after cold acclimation, suggesting that the three $C B F$ genes together are essential for cold acclimation (Zhao et al., 2016). Recently, ICE1 (inducer of CBF expression 1) was identified as an upstream transcription factor regulating the transcription of $C B F$, and its overexpression activated the expression of $C B F$ regulon under cold condition and improved freeze tolerance of the transgenic plants (Chinnusamy et al., 2003). However, transcriptome profiling experiments showed that the number of CBF regulon gene accounts only $6.5 \%$ of the total number of $C O R$ genes, suggesting that other transcription factors are also involved in the regulation of $C O R$ genes and the low-temperature regulatory network beyond the CBF pathway is complex and highly interconnected (Park et al., 2015). Recently, many other $C O R$ genes were identified from $C B F$-independent pathways. For example, SCOF-1 encodes a cold-inducible zinc finger protein from soybean, and $O s m y b 4$ encodes a member of $M Y B$ transcription factor from rice, these genes also contributed to cold tolerance in plants (Kim et al., 2001; Vannini et al., 2004).

Thellungiella salsuginea also named as T. halophile or salt cress, is a close relative of Arabidopsis. Compare to Arabidopsis,
T. salsuginea exhibits higher tolerance to cold, and it could complete its life cycles at $5^{\circ} \mathrm{C}$, and could survive at extreme low temperature of $-21^{\circ} \mathrm{C}$ after cold acclimation (Griffith et al., 2007). Thus, Thellungiella was proposed as an appropriate model to investigate the molecular mechanisms of plant adapted to cold stress (Griffith et al., 2007; Amtmann, 2009). To illustrate how Thellungiella adapts to low-temperature, cold regulated genes were identified from both the mRNA and protein levels. In a survey of 3,628 Thellungiella cDNAs, 76 cold induced transcripts including COR47, ERD10, and COR15b were identified using microarray methods (Wong et al., 2006). Northern blot analysis demonstrated that some cold response genes (CBF1, COR15a, and COR47) from Arabidopsis were also induced in Thellungiella (Griffith et al., 2007). Two-dimensional electrophoresis (2-DE) approach was used in Thellungiella, and found 66 protein spots were significantly affected by cold in Thellungiella rosette leaves (Gao et al., 2009). These studies provided useful clues for understanding the mechanism of cold tolerance in Thellungiella. However, due to the limited genomic sequences, these studies fail to provide a comprehensive interpretation of the transcriptomic changes of Thellungiella in response to cold. To gain insight into the molecular networks underlying Thellungiella cold tolerance, more comprehensive genome-wide gene expression profiling studies are required.

Recently, the whole genome sequence of Thellungiella was completed, which provides new opportunity to understand the cold tolerance mechanism in Thellungiella (Dassanayake et al., 2011; Wu et al., 2012). In this study, we carried out genome-wide analysis of gene expression in roots and leaves of Thellungiella under cold treatment using RNA-seq technology. The aim of the study is to identify cold responsive genes and biological pathways that may contribute to cold tolerance in Thellungiella. We identified thousands of cold-responsive genes and provided an overall picture of the regulatory network in response to cold stress in Thellungiella. These cold-responsive genes could be targeted as potential candidates for further functional validation, and have potential application value for increasing cold tolerance in crops.

\section{RESULTS AND DISCUSSION}

\section{High Throughput Sequencing and Gene Expression Profiles}

To gain the profiles of gene expression in Thellungiella under cold condition, eight cDNA libraries were constructed using roots and leaves under normal (control) and low temperature (cold) for $24 \mathrm{~h}$, respectively. The cDNA libraries were sequenced by Illumina Hiseq2000 platform using the paired end method. After removing low quality, $\mathrm{N}$-containing and adaptor-contaminated reads, a total of $96,305,447$ clean reads were generated, with an average of $\sim 12$ million reads per libraries (Table 1). Approximately $90 \%$ reads from leaves and $85 \%$ reads from roots were mapped to the Thellungiella reference genome, and about $7 \%$ reads from leaves and 3.5\% reads from roots were mapped to multiple regions, respectively (Table 1 ). The RNA-seq raw 


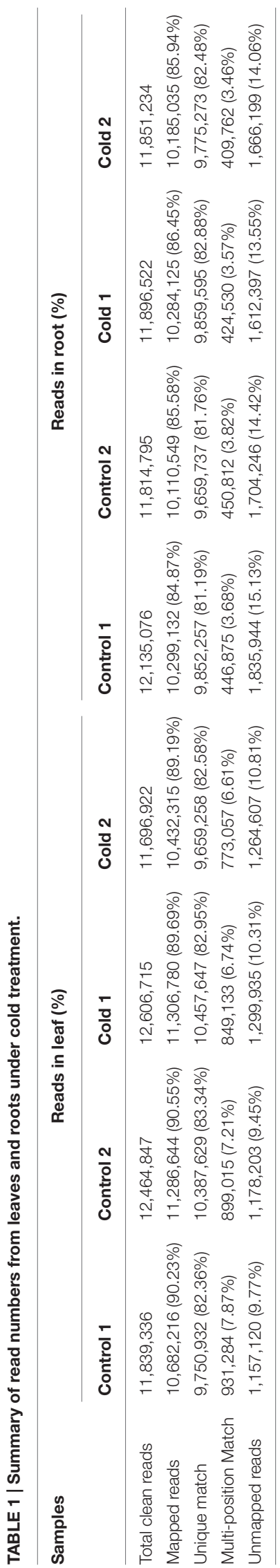

sequencing data from this study have been submitted in the SRA database $^{1}$ under BioProjects: PRJNA377594 (SRA: SRP101369).

Among the 29,284 genes deposited in Thellungiella genome database, $22,414(76.5 \%)$ genes were detected in the control and cold-treated libraries. A total of 2,782 and 1,430 differentially expressed genes (DEGs) were identified from leaves and roots, respectively (Figure 1 and Supplementary Tables S1, S2). Under cold treatment, 579 and 1,691 genes were up-regulated, 851 and 1,091 genes were down-regulated in roots and leaves, respectively (Figure 1). Our results demonstrated that the expression patterns of the majority of DEGs were different in roots and leaves. For example, among the 1,691 up-regulated genes in leaves, only 269 (15.9\%) were also induced in roots (Figure 2). Interestingly, some genes showed the opposite expression trend in roots and leaves upon cold treatment. For example, fifteen DEGs were up-regulated in root, but down-regulated in leaves. There were 68 DEGs down-regulated in root, but up-regulated in leaves (Figure 2). Moreover, 463 DEGs were with the same expression trend in roots and leaves, including 269 up-regulated and 194 down-regulated DEGs (Figure 2 and Supplementary Table S1).

\section{Functional Analysis of DEGs}

The DEGs were characterized by the assignment of gene ontology (GO) terms using Blast2GO (Conesa et al., 2005) program. GO enrichment analysis showed 241 and 374 GO terms were significant enriched in root and leaf, respectively (Supplementary Table S3). Then, we employed WEGO web-based tool to visualize the biological process, molecular function and cellular component main categories (Figure 3). In the cellular component category, the terms of "cell", "cell part", "organelle", and "membrane" were enriched, implying the potential contribution of cell and cell structure in the process of Thellungiella response to cold condition. For the category of molecular function, "binding", "catalytic activity" and "nucleic acid binding transcription factor activity" were the top terms. The most abundant terms of

${ }^{1}$ https://www.ncbi.nlm.nih.gov/sra/

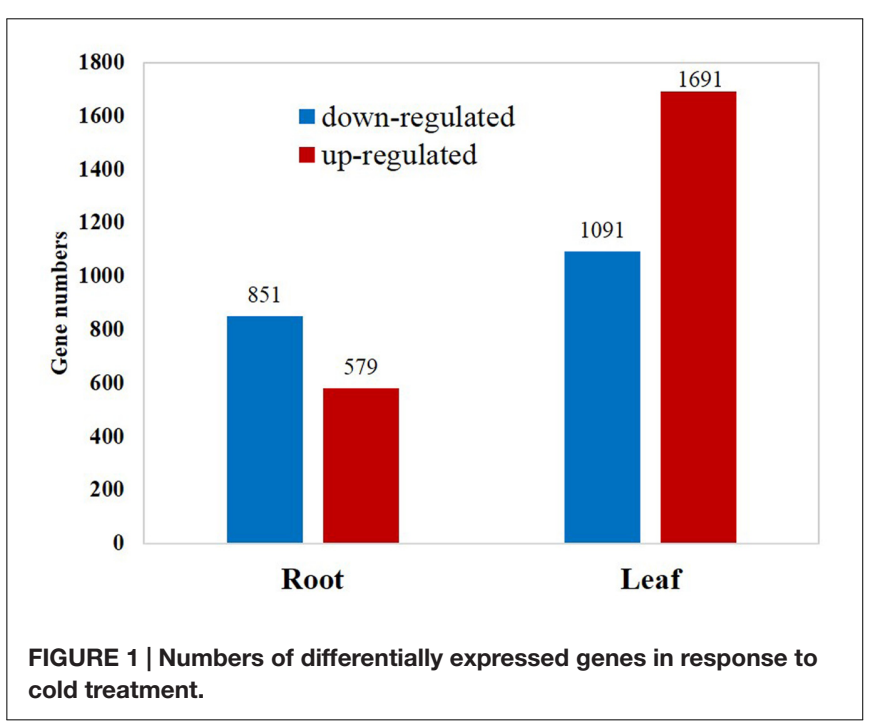



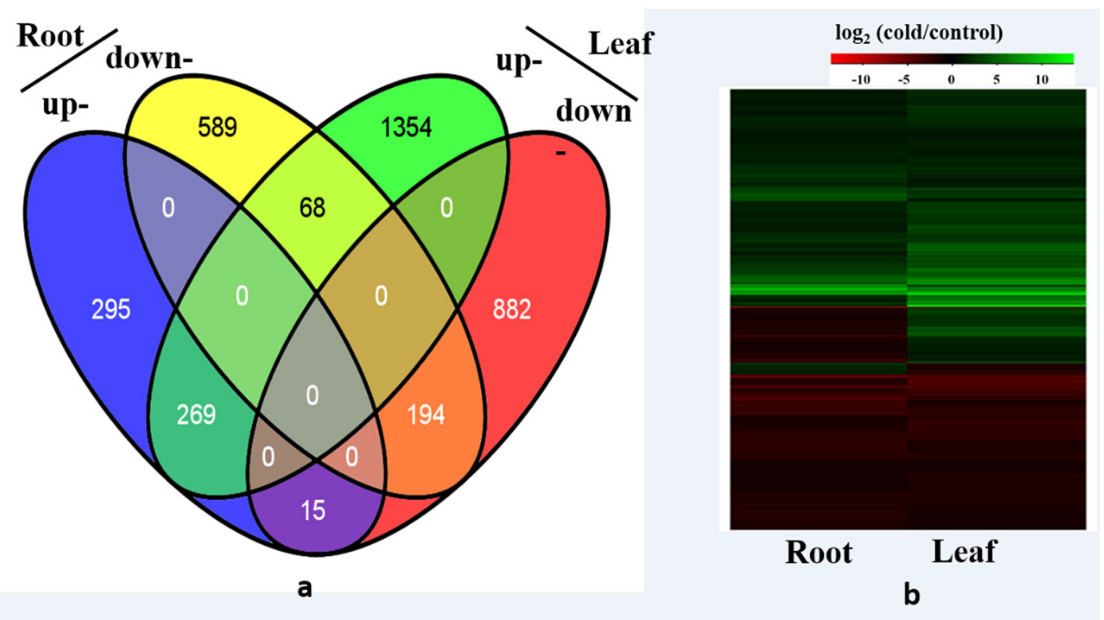

FIGURE 2 | Differentially expressed genes analysis. (a) Venn diagram demonstrated the common and specific differentially expressed genes (DEGs) in roots and leaves, (b) Heat map demonstrated the expression profile DEGs in roots and leaves.

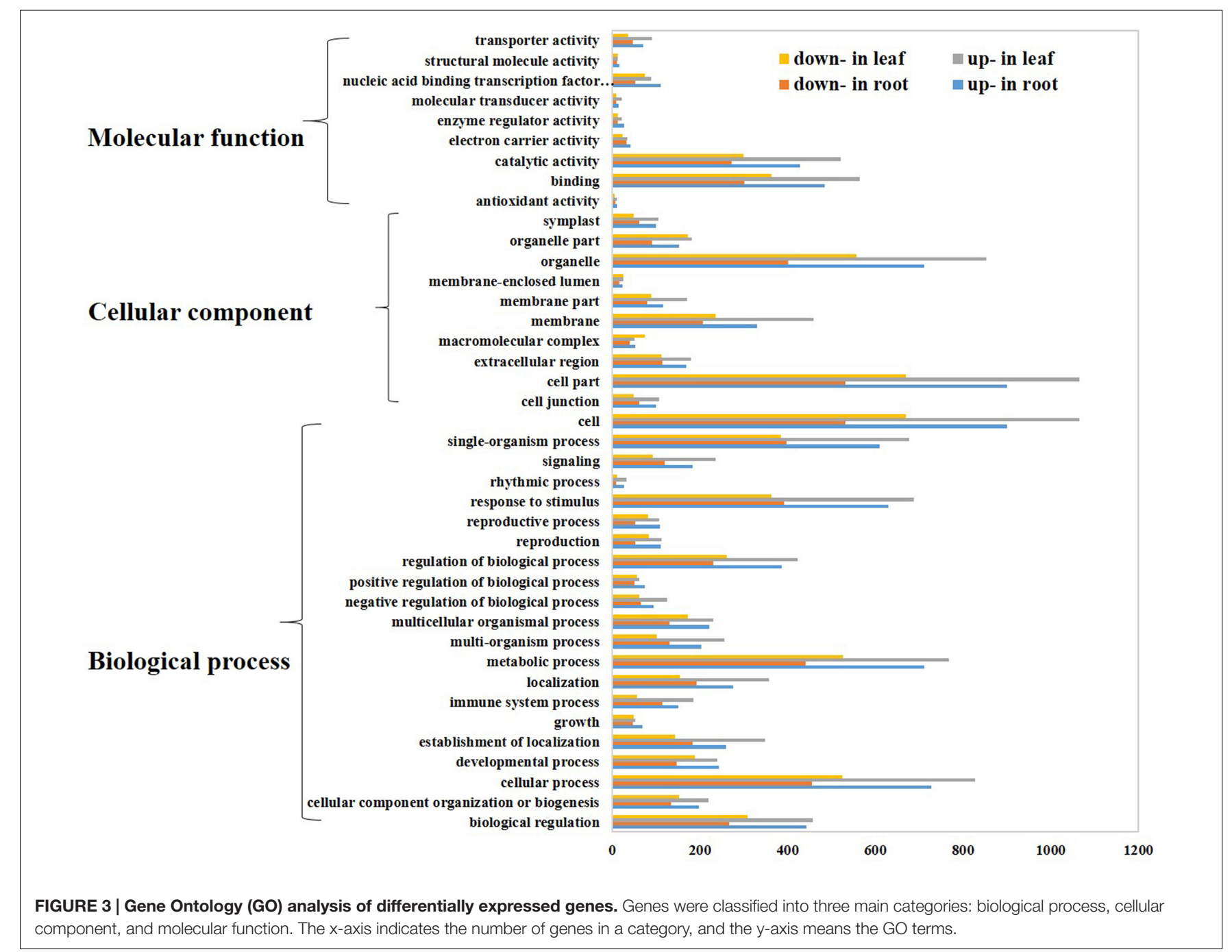


biological process were "cellular process", "metabolic process", "response to stimulus" and "single-organism process", suggesting a high degree of metabolic activity changes upon cold treatment (Figure 3).

In order to obtain more biological information for understanding the molecular mechanism and regulatory network of Thellungiella cold tolerance, KEGG enrichment pathway analysis was performed. By applying a cut-off criterion of $Q$-value $<0.05$ and $P$-value $<1 \mathrm{E}^{-05}$, the result showed that ten and fifteen pathways were significantly enriched from roots and leaves, respectively (Table 2). Previous studies demonstrated that many metabolic changes for enhancing freeze tolerance in Arabidopsis, such as increasing accumulation of soluble sugars and other compatible osmolytes (Wanner and Junttila, 1999). We found six pathways, "biosynthesis of secondary metabolites", "metabolic pathways", "nitrogen metabolism", "tryptophan metabolism", "cysteine and methionine metabolism", and "sulfur metabolism" were enriched both in roots and leaves in response to cold. Interestingly, all these pathways were involved in a particular metabolic process, suggesting that the metabolic process was activated via cold treatment (Table 2). Proline is one of the most effective organic osmolytes in plants, and there is a positive correlation between proline accumulation and plant stress tolerance. A number of studies showed that proline played beneficial roles in plants when exposed to cold condition (Hayat et al., 2012). Previous studies demonstrated that Thellungiella contained higher levels of proline than Arabidopsis under nonstressed condition (Kant et al., 2006). Twenty-six genes involved in "Arginine and proline metabolism" pathway were found to be regulated by cold in our study. Most of these DEGs (18 of 26) were up-regulated, suggesting that these cold induced genes might promote the accumulation of proline in Thellungiella to enhance cold tolerance.

The "biosynthesis of unsaturated fatty acids" pathway was also enriched in Thellungiella leaves. A total of 22 genes involved in this pathway were affected by low temperature (Table 2). Previous study suggested that membrane lipid composition, especially the concentration of unsaturated fatty acid, is highly correlated with plant freezing tolerance (Thomas et al., 2012). The expression regulation of genes for unsaturated fatty acid synthesis might be a key factor contributing to cold tolerance in Thellungiella.

We observed that the pathways related to biosynthesis of phenylpropanoid and flavonoid were enriched in roots of Thellungiella. In addition, "Photosynthesis - antenna proteins" and "Photosynthesis" were enriched. Most of the DEGs in "Photosynthesis-antenna proteins" and "Photosynthesis" pathways were down-regulated in leaves, suggesting the adverse effect of low temperature on photosynthetic carbohydrate metabolism and photochemical reaction (Supplementary Table S2).

TABLE 2 | The top enriched pathways of DEGs in roots and leaves.

\begin{tabular}{|c|c|c|c|c|c|}
\hline Pathway & DEGs (\%) & All genes & $P$-value & Q-value & Pathway ID \\
\hline \multicolumn{6}{|c|}{ Root: 1043 DEGs with KEGG pathway annotation } \\
\hline Biosynthesis of secondary metabolites & $217(20.81 \%)$ & $1727(11.11 \%)$ & 2.99E-21 & $3.52 \mathrm{E}-19$ & ko01110 \\
\hline Metabolic pathways & $326(31.26 \%)$ & $3484(22.41 \%)$ & $4.96 \mathrm{E}-12$ & $2.93 \mathrm{E}-10$ & ko01100 \\
\hline Nitrogen metabolism & $22(2.11 \%)$ & $76(0.49 \%)$ & 2.73E-09 & $1.07 \mathrm{E}-07$ & ko00910 \\
\hline Phenylpropanoid biosynthesis & $49(4.70 \%)$ & $306(1.97 \%)$ & $1.04 \mathrm{E}-08$ & $3.08 \mathrm{E}-07$ & ko00940 \\
\hline Flavonoid biosynthesis & $33(3.16 \%)$ & $172(1.11 \%)$ & $3.47 \mathrm{E}-08$ & $8.20 \mathrm{E}-07$ & ko00941 \\
\hline Phenylalanine metabolism & $28(2.68 \%)$ & $151(0.97 \%)$ & $7.72 \mathrm{E}-07$ & $1.52 \mathrm{E}-05$ & ko00360 \\
\hline Tryptophan metabolism & $22(2.11 \%)$ & $127(0.82 \%)$ & $3.48 \mathrm{E}-05$ & $5.87 \mathrm{E}-04$ & ko00380 \\
\hline Cysteine and methionine metabolism & $21(2.01 \%)$ & $123(0.79 \%)$ & $6.48 \mathrm{E}-05$ & 8.08E-04 & ko00270 \\
\hline Sulfur metabolism & $13(1.25 \%)$ & $56(0.36 \%)$ & $6.51 \mathrm{E}-05$ & 8.08E-04 & ko00920 \\
\hline Galactose metabolism & $16(1.53 \%)$ & $80(0.51 \%)$ & $6.85 E-05$ & 8.08E-04 & ko00052 \\
\hline \multicolumn{6}{|c|}{ Leaf: 2419 DEGs with KEGG pathway annotation } \\
\hline Biosynthesis of secondary metabolites & 408 (16.87\%) & 1727 (11.11\%) & $7.46 \mathrm{E}-21$ & $9.55 \mathrm{E}-19$ & ko01110 \\
\hline Metabolic pathways & $687(28.40 \%)$ & $3484(22.41 \%)$ & 3.31E-14 & $2.12 \mathrm{E}-12$ & ko01100 \\
\hline Photosynthesis - antenna proteins & $16(0.66 \%)$ & $27(0.17 \%)$ & $2.62 \mathrm{E}-07$ & $1.12 \mathrm{E}-05$ & ko00196 \\
\hline Nitrogen metabolism & $29(1.20 \%)$ & $76(0.49 \%)$ & $1.40 \mathrm{E}-06$ & 4.47E-05 & ko00910 \\
\hline Photosynthesis & $30(1.24 \%)$ & $81(0.52 \%)$ & $1.92 \mathrm{E}-06$ & 4.92E-05 & ko00195 \\
\hline Pyruvate metabolism & $40(1.65 \%)$ & $125(0.80 \%)$ & $3.22 \mathrm{E}-06$ & $6.87 \mathrm{E}-05$ & ko00620 \\
\hline Biosynthesis of unsaturated fatty acids & $22(0.91 \%)$ & $53(0.34 \%)$ & 5.16E-06 & $9.44 \mathrm{E}-05$ & ko01040 \\
\hline Valine, leucine and isoleucine biosynthesis & $17(0.70 \%)$ & $37(0.24 \%)$ & $1.20 \mathrm{E}-05$ & $1.92 \mathrm{E}-04$ & ko00290 \\
\hline Sulfur metabolism & $22(0.91 \%)$ & $56(0.36 \%)$ & 1.49E-05 & $2.12 \mathrm{E}-04$ & ko00920 \\
\hline Amino sugar and nucleotide sugar metabolism & $47(1.94 \%)$ & $167(1.07 \%)$ & $2.30 \mathrm{E}-05$ & $2.94 \mathrm{E}-04$ & ko00520 \\
\hline Glyoxylate and dicarboxylate metabolism & $26(1.07 \%)$ & $74(0.48 \%)$ & 2.70E-05 & $3.14 \mathrm{E}-04$ & ko00630 \\
\hline Cysteine and methionine metabolism & $37(1.53 \%)$ & $123(0.79 \%)$ & $3.48 \mathrm{E}-05$ & $3.71 \mathrm{E}-04$ & ko00270 \\
\hline Circadian rhythm - plant & $45(1.86 \%)$ & $162(1.04 \%)$ & 4.81E-05 & $4.74 \mathrm{E}-04$ & ko04712 \\
\hline Tryptophan metabolism & $37(1.53 \%)$ & $127(0.82 \%)$ & $7.41 \mathrm{E}-05$ & $6.44 \mathrm{E}-04$ & ko00380 \\
\hline Glycine, serine and threonine metabolism & $29(1.20 \%)$ & $91(0.59 \%)$ & 7.55E-05 & $6.44 \mathrm{E}-04$ & ko00260 \\
\hline
\end{tabular}


In Arabidopsis, environmental temperature affected the expression of clock component related genes, such as timing of cab expression 1 (TOC1), GIGANTEA (GI), circadian clock associated 1 (CCA1), and late elongated hypocotyl (LHY). Here, our results demonstrated that the pathway of "circadian rhythm - plant" was enriched in Thellungiella after cold treatment. The expression of many genes involved in circadian rhythm including LHY, CCA1, TOC1 and GI were also induced or inhibited in Thellungiella upon cold treatment (Table 2 and Supplementary Table S2).

\section{Validation of RNA-Seq Results Using Quantitative Real-Time PCR}

To validate the RNA-seq data, quantitative real-time PCR (qRTPCR) was performed for 25 genes with different expression levels and functional assignments (Supplementary Table S4). Among them, seven genes were significantly induced upon cold treatment both in roots and leaves. Two of these genes encoded salt and low temperature response protein, one encoded dehydrin. A putative phosphatidylethanolamine-binding protein gene, and three genes with unknown function were also selected for qRT-PCR validation. Other ten selected genes were downregulated both in roots and leaves. These genes included one aquaporin TIP2-1, one NAC domain protein, one stressinduced stil-like protein coding genes and several genes with unknown function. In addition, eight genes that down-regulated in roots but up-regulated in leaves were also selected for qRT-PCR analysis. These genes included O-methyltransferase family protein, peroxidase, cytochrome $\mathrm{P} 450$, leucine-rich repeat receptor-like protein kinase, MYB and zine finger AN1 domaincontain protein coding genes (Supplementary Table S4). The
qRT-PCR results of these 25 genes in leaves were in agreement with the RNA-seq data. The relative expression level $\left(\log _{2}\right.$ cold/control) estimated by RNA-seq and qRT-PCR were strongly correlated $\left(R^{2}=0.9695\right)$ in leaves (Supplementary Table S4 and Figures 4, 5). In roots, the majority of genes (23 of 25) showed similar expression patterns except Thhalv10004977m and Thhalv10002333m, which were down-regulated in RNA-seq, but up-regulated in qRT-PCR. In roots, the Pearson's coefficient was 0.7664 which was lower than that in leaves (Figure 4 and Supplementary Figure S2).

\section{Cold Related Transcription Factors}

Studies showed that transcription factors played important roles in plant response to low temperature and other adverse stresses (Singh et al., 2002; Chen and Zhu, 2004; Agarwal P.K. et al., 2006). We observed that many transcript factors were response to cold in Thellungiella. Among the 1,430 DEGs in roots, 159 genes (11.1\%) were annotated as different types of transcription factors (Table 3). About half of these transcription factor genes (74 of 159) were up-regulated, the rest 85 genes were down-regulated. According to functional annotation, these transcription factors were classified into 18 categories, such as abscisic acid responsive, NAC, Zinc finger domain, AP2, MYB, bHLH and WRKY etc. The transcription factors Zinc finger domains (27 genes, 17.0\%), ethylene responsive (11 genes, 6.9\%) and calcium ion binding (10 genes, $6.3 \%$ ) were the three major families of the cold-regulated transcription factors in roots.

Among the 2,782 DEGs in leaves, 232 (8.3\%) were transcription factors, including 131 up-regulated and 101 down-regulated genes. These transcription factors were classified into 18 categories (Table 3). Among these differential expressed
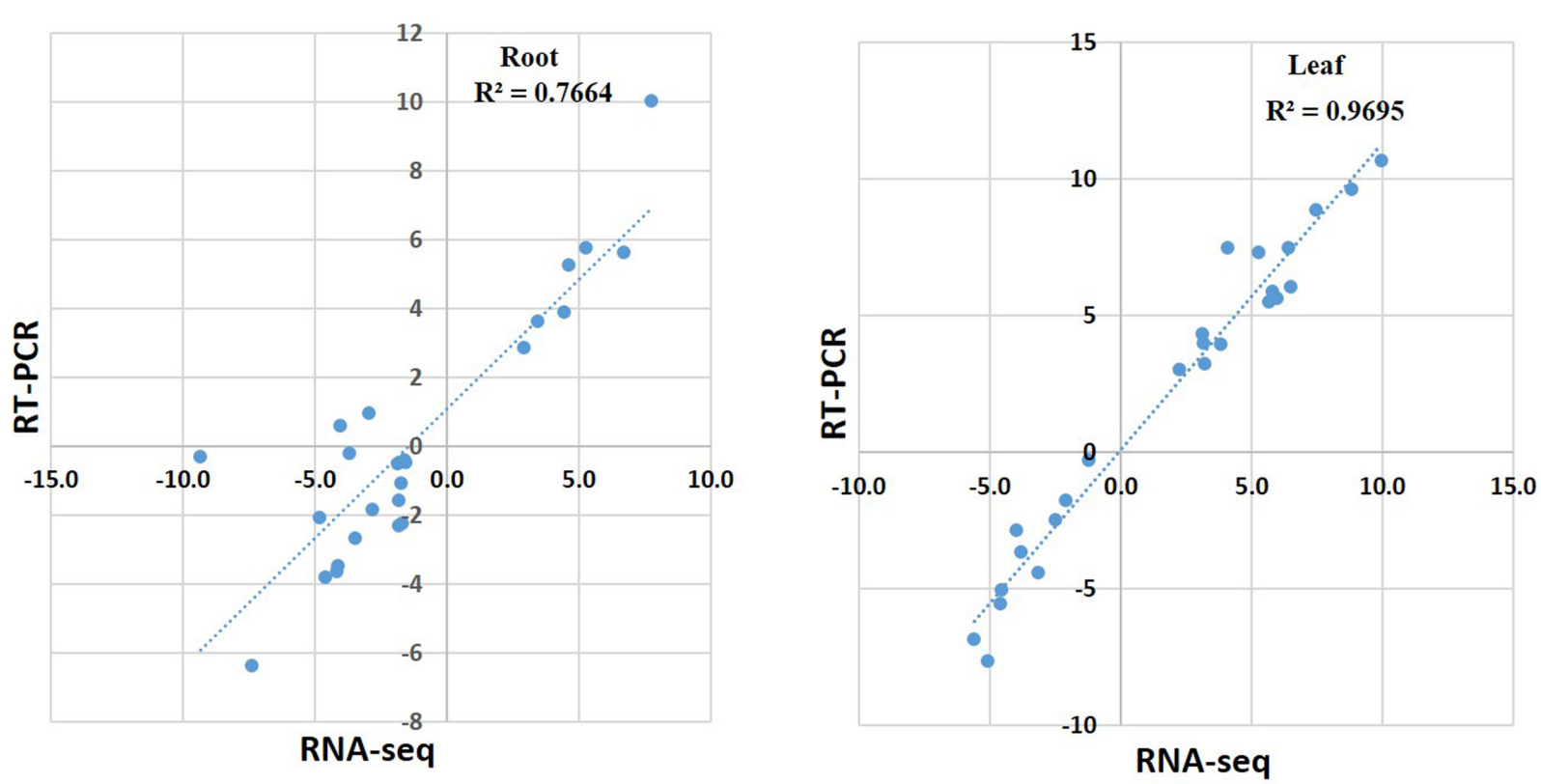

FIGURE 4 | Pearson's correlation of RNA-seq and qRT-PCR results. qRT-PCR validation of DEGs in roots and leaves under cold condition. The correlation of the fold change analyzed by RNA-Seq ( $x$-axis) with data obtained using qRT-PCR ( $y$-axis). 
TABLE 3 | Cold-regulated transcription factors.

\begin{tabular}{|c|c|c|c|c|c|c|}
\hline \multirow[t]{2}{*}{ Category } & \multicolumn{3}{|c|}{ Number of transcription factors in root } & \multicolumn{3}{|c|}{ Number of transcription factors in leaf } \\
\hline & Total & Up-regulated & Down-regulated & Total & Up-regulated & Down-regulated \\
\hline Abscisic acid responsive & 4 & 3 & 1 & 1 & 1 & 0 \\
\hline AP2 & 1 & 0 & 1 & 2 & 1 & 1 \\
\hline Auxin-responsive & 4 & 2 & 2 & 5 & 2 & 3 \\
\hline Basic leucine zipper & 3 & 1 & 2 & 1 & 0 & 1 \\
\hline $\mathrm{bHLH}$ & 4 & 0 & 4 & 4 & 1 & 3 \\
\hline BTB and TAZ domain & 1 & 1 & 0 & 1 & 0 & 1 \\
\hline Calcium ion binding & 10 & 0 & 10 & 6 & 5 & 1 \\
\hline Ethylene responsive & 11 & 4 & 7 & 5 & 3 & 2 \\
\hline F-box & 5 & 1 & 4 & 5 & 0 & 5 \\
\hline Heat shock protein & 1 & 0 & 1 & 9 & 5 & 4 \\
\hline Homeobox-leucine zipper & 1 & 1 & 0 & 4 & 0 & 4 \\
\hline Leucine-rich repeat & 6 & 1 & 5 & 7 & 2 & 5 \\
\hline MADS-box & 2 & 2 & 0 & 1 & 0 & 1 \\
\hline MYB & 6 & 3 & 3 & 10 & 3 & 7 \\
\hline NAC-domain & 2 & 1 & 1 & 9 & 7 & 2 \\
\hline WRKY & 3 & 0 & 3 & 12 & 11 & 1 \\
\hline Zinc finger domain & 27 & 15 & 12 & 43 & 33 & 10 \\
\hline Ulassification & 68 & 39 & 29 & 107 & 57 & 50 \\
\hline Total & 159 & 74 & 85 & 232 & 131 & 101 \\
\hline
\end{tabular}

transcription factors in leaves, Zinc finger domain, WRKY and MYB are enriched. Heat shock proteins (HSPs) were implicated in plant heat stress tolerance (Nover et al., 2002). Here, we found five HSPs were up-regulated and four HSPs were down-regulated upon cold treatment. WRKY transcription factors played important roles in plant responses to biotic and abiotic stress (Eulgem et al., 2000). Twelve differentially expressed WRKY transcription factor genes were identified in leaves, and eleven of them were up-regulated. NAC transcription factors were involved in many aspects of plant growth and development, and response to abiotic stress (Nuruzzaman et al., 2013). In our RNA-seq data, nine and three differentially expressed NAC transcription factor genes were identified in leaves and roots, respectively. These results indicated that HSF, WRKY and NAC transcription factors were involved in plant responses to various stresses, and suggested that cold stress might share common molecular mechanism with other abiotic stresses. In addition, we noticed that the expression of ten calcium ion binding transcription factors was all suppressed in roots upon cold treatment, while most of them were induced in leaves, implying that these genes might be functioning in different ways in roots and leaves (Table 3 and Supplementary Table S1). These results suggested the existence of differences in cold responsive regulatory networks between roots and leaves in Thellungiella.

Previous studies showed that some MYB transcription factors were involved in cold stress tolerance, such as AtMYB15 in Arabidopsis (Agarwal M. et al., 2006), MYB55 and OsMYBS3 in rice (Su et al., 2010; Elkereamy et al., 2012). In our data, the expression of six and ten $M Y B$ genes was altered after cold treatment in roots and leaves, respectively (Table 3 and Supplementary Tables S1, S2). Interestingly, Thhalv10008152m encoding a MYB transcription factor displayed opposite expression trend in roots and leaves. In roots, the expression of this gene was inhibited by cold treatment, while it was induced in leaves (Figure 5).

Basic helix-loop-helix (bHLH)-type transcription factors played important regulatory roles in diverse biological processes in plants (Toledoortiz et al., 2003). The latest evidences showed that $b H L H$ transcription factor acted as positive regulators of CBF-pathway and conferred cold tolerance in plants (Feng et al., 2012; Peng et al., 2013; Xu et al., 2014). We found that the expression of four and four $b H L H$ genes was altered after cold treatment in Thellungiella roots and leaves, respectively (Table 3 and Supplementary Tables S1, S2). However, only one of these $b H L H$ gene (Thhalv10025656m) was induced by cold, and the others were down-regulated in response to cold treatment. Functional annotation showed that Thhalv10025656m was homologous of bHLH69 of Arabidopsis, and named as TsbHLH69. In Arabidopsis, bHLH69 contributed to the regulation of circadian periodicity by reducing the expression of $L H Y$ and TOC1 (Hanano et al., 2008; Harmer, 2009). These data suggested that the cold-induced TsbHLH69 might participate in the regulation of rhythm of Thellungiella under cold condition.

\section{Cold Regulation of Genes Related to Plant Hormone Biosynthesis and Signaling}

In this study, we found that the expression of many genes related to plant hormone biosynthesis and signaling were altered upon cold treatment (Supplementary Tables S1, S2). A total of 134 DEGs were related to plant hormone biosynthesis and signaling 

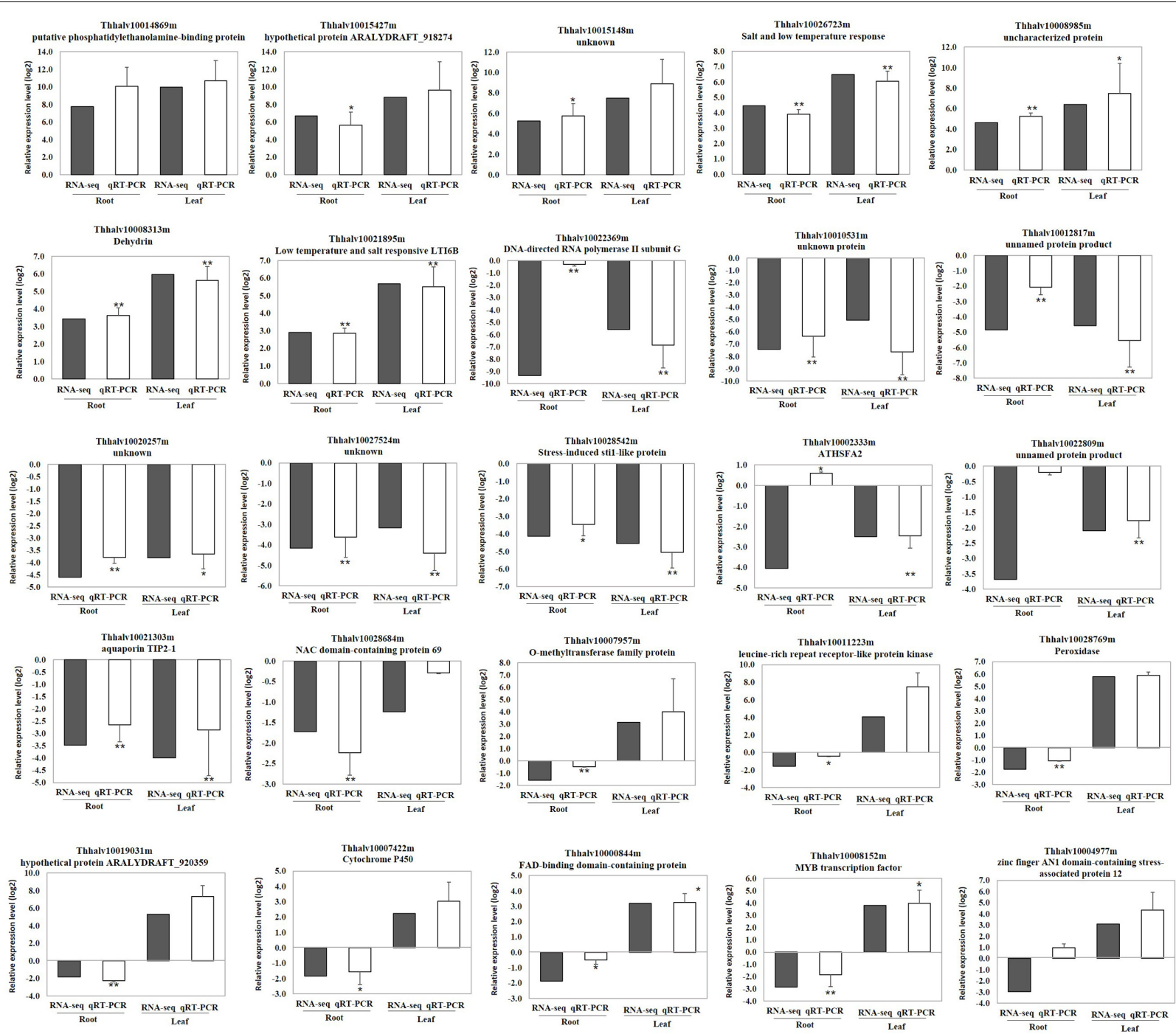

FIGURE 5 | Validation of gene expression by qRT-PCR. Error bars indicate \pm SE obtained from three biological repeats. Student's $t$-test was performed to analyze the changes in the gene expression after treated with cold. **denotes the $p$-value $<0.01$ and *denotes the $p$-value $<0.05$.

in leaves, 71 of which were up-regulated and 73 of which were down-regulated. DELLA, an key factor in gibberellins (GA) signaling, was also involved in the signal transduction of other hormones suggesting that DELLA functions as a modulator of plant development and response to stresses (Achard et al., 2003; Willige et al., 2007). Studies showed that DELLA contributed to CBF1-induced cold acclimation and was considered as components of CBF1-mediated cold stress response (Achard et al., 2008). We found the expression of a DELLA encoding gene (Thhalv10015535m) was slightly induced in both roots (1.40 fold) and leaves (1.39 fold) upon cold treatment. Meanwhile, gibberellin 2-oxidase (Thhalv10008179m), an enzyme inactivating the bioactive gibberellins (GAs), was significantly induced (2.6-fold) (Supplementary Table S2). These results implied that GA metabolism and signaling might contribute to cold stress tolerance in Thellungiella.

\section{Other Cold-Regulated (COR) Genes}

Studies demonstrated that the expression of COR genes was strongly induced after plants were shifted to cold temperature (Hajela et al., 1990; Griffith et al., 2007). RNA-seq results revealed five COR genes including COR27, COR47, and three COR15 were dramatically induced in both roots and leaves under cold treatment (Table 4). Interestingly, we found that the expression levels of all three COR15 increased by 1,000-fold in leaves after cold treatment. Among all these COR genes, COR15 represented the most induced genes, whose expression level increased more than 8,000-fold to compare with the control. Sequence alignment results showed that the DNA sequences of these three COR15 genes were highly similar, implying these genes might have the same functions in cold tolerance (Supplementary Figure S1). The COR47 gene encoding a member of dehydrin has been isolated in several plant species. Previous studies showed that 
TABLE 4 | The expression profile of cold-regulated genes.

\begin{tabular}{|c|c|c|c|c|c|c|}
\hline \multirow[t]{2}{*}{ Gene } & \multirow[t]{2}{*}{ Annotation } & \multicolumn{4}{|c|}{ Relative expression level (cold/control) } & \multirow[t]{2}{*}{ Expression trend } \\
\hline & & $\log 2$ (leaf) & $Q$-value (leaf) & $\log 2$ (root) & Q-value (root) & \\
\hline Thhalv10017395m & Cold response protein (COR15) & 13.40 & 1.000 & 3.36 & 0.830 & Up \\
\hline Thhalv10017393m & Cold response protein (COR15) & 10.73 & 0.961 & 7.44 & 0.540 & Up \\
\hline Thhalv10017394m & Cold response protein (COR15) & 10.22 & 0.943 & 8.11 & 0.932 & Up \\
\hline Thhalv10003264m & COR27 & 9.26 & 0.979 & 1.18 & 0.809 & Up \\
\hline Thhalv10008706m & $\mathrm{COR} 47$ & 6.80 & 0.999 & 6.54 & 0.998 & Up \\
\hline Thhalv10027405m & $C B F / D R E B 1$ & 2.85 & 0.89 & 1.61 & 0.90 & Up \\
\hline
\end{tabular}

the promoter region of COR47 contained C-repeat, dehydrationresponsive element, low temperature-responsive element (CRT/DRE/LTREs) and ABA regulatory element (ABRE). COR47 was the downstream gene of $C B F / D R E B 1$, and $C B F / D R E B 1$ binds to the promoter of $C O R 47$ to induce its expression (Jaglo-Ottosen et al., 1998). Functional annotation and sequence alignment showed that Thhalv10027405m, a TsCBF/DREB1 gene, was a homolog of Arabidopsis CBF1/2/3. RNA-seq data revealed that Thhalv10027405m was up-regulated in both roots and leaves under cold treatment (Table 4 and Supplementary Table S2). The expression profiles of TsCBF/DREB1 and COR47 in Thellungiella was consistent with their counterparts in Arabidopsis, suggesting that $C B F / D R E B 1$ and $C O R 47$ genes might play important roles in $D R E B 1 / C B F$-dependent cold signaling pathway in Thellungiella.

In addition, our RNA-seq data indicated that there might be more genes involved in DREB1/CBF pathway in Thellungiella. For example, we found $D R E B 2 B$ gene (Thhalv10021161m) was up-regulated in leaves under cold treatment (Supplementary Table S2). In addition to COR47 gene, we observed other two dehydrin genes, Thhalv10010821m and Thhalv10008313m were also induced by cold treatment (Figure 5 and Supplementary Table S2).

In Arabidopsis, INDUCER OF CBF EXPRESSION 1 (ICE1) could induce $C B F 3$ expression by binding to its promoter. It was an important upstream regulatory factor in $D R E B 1 / C B F$ signaling pathway (Chinnusamy et al., 2003). In Thellungiella, we observed that the expression of ICE1 (Thhalv10004059m) were up-regulated in roots upon cold treatment (1.57-fold). However, the expression level of ICE1 was similar in cold treated and control samples in leaves. These results indicated that the function of ICE1 might be different in Arabidopsis and Thellungiella.

Aquaporins (or water channel proteins) played a crucial role in plant water relations. According to their distinct sub-cellular localization, aquaporins could be divided into four subgroups, the tonoplast intrinsic proteins (TIPs), plasma membrane intrinsic proteins (PIPs), Nod26-like intrinsic membrane proteins (NIPs) and small basic intrinsic proteins (SIPs). TIPs were abundantly expressed in vacuole (Maurel, 2007) and played an important role in maintenance of the intracellular space by controlling the water influx in vacuole (Leitão et al., 2014). A member of tonoplast intrinsic proteins GhTIP1;1 was responsive to cold stress and contributed to freezing-tolerance in cotton (Li D.D. et al., 2009). In the current study, five cold regulated aquaporin genes including three TIPs, one PIPs and one NIPs were identified. RNA-seq and qRT-PCR results demonstrated that TIP2-1 (Thhalv10021303m) was significantly down-regulated in both roots and leaves when the plants were under cold treatment. Another two TIP genes, Thhalv10011730m (also annotated as TIP2-1) and Thhalv10002084m (TIP4-1), were also significantly down-regulated upon cold treatment. These results suggested that the decreased expression of TIP genes might be beneficial to reduce water in/out of vacuole which is important for maintaining the stability of the cells in cold condition (Figure 5 and Supplementary Tables S1, S2). Moreover, we observed that the expression of PIP2-7 (Thhalv10025940m) was significantly decreased, suggesting that the water in/out of the cell might be reduced.

\section{CONCLUSION}

In this study, we compared the transcriptome of Thellungiella roots and leaves in response to cold treatment using RNA-seq. We identified a number of cold-responsive genes which were involved in different pathways closely related to environmental adaptation and other biological processes, suggesting the complex responses of Thellungiella toward cold stress (Figure 6). Our findings provided an overall picture of the regulatory network in response to cold stress in Thellungiella. These coldresponsive genes could be targeted as potential candidates for further functional validation, and have potential application value for increasing cold tolerance in crops.

\section{MATERIALS AND METHODS}

\section{Plant Materials}

Seeds of T. salsuginea (Shandong ecotype) were surface-sterilized and plated on 1/2 MS-agar plates for synchronize germination at $4^{\circ} \mathrm{C}$ for a week. The plates were moved to growth chamber with $16 \mathrm{~h}$ light at $26^{\circ} \mathrm{C}$ with light intensity of $3000 \mathrm{~lx}$ and $8 \mathrm{~h}$ dark at $22^{\circ} \mathrm{C}$. Seven-day-old seedlings were transferred to soil for 5 weeks. For cold treatment, seedlings were exposed to a growth chamber under a $16 / 8 \mathrm{~h}$ light/dark regime at $8 / 4^{\circ} \mathrm{C}$ for $24 \mathrm{~h}$. For the control, the seedlings were grown at $22 / 16^{\circ} \mathrm{C}$. Leaves and roots of control and cold treated seedlings were collected, frozen immediately in liquid nitrogen, and stored at $-80^{\circ} \mathrm{C}$. To minimize the plant to plant variation, nine individuals were used as one biological replicate, the tissue from 


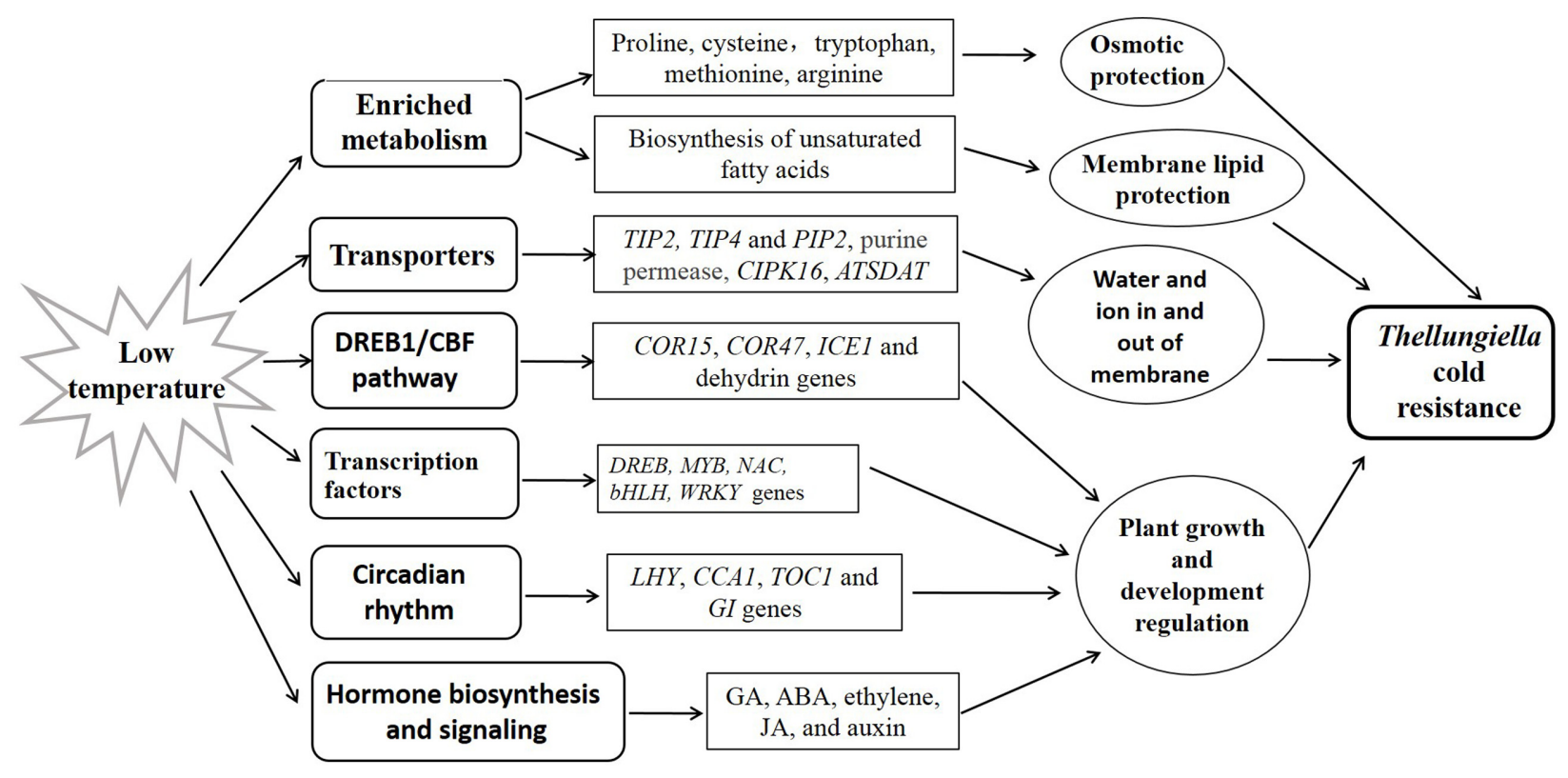

FIGURE 6 | Simple model deduced based on the transcriptome data.

nine individuals were pooled into one independent biological replicate. For both leaves and roots, three biological replicates were prepared.

\section{RNA Isolation, cDNA Library Construction, and Sequencing}

Total RNA was isolated from leaves and roots using RNAiso Reagent (Takara, China), and treated with DNase I (Takara, China) to remove the contaminated genomic DNA according to the manufacturer's protocols. RNA quality was detected by electrophoresis on $1.0 \%$ agarose gels and NanoDrop. The mRNA was enriched and cleaved into short fragments (about $200 \mathrm{nt})$. The mRNA fragments were used as templates to synthesize the first strand cDNA using random hexamerprimer. The first strand cDNA was further incubated with DNA polymerase I, buffer, dNTPs and RNase $\mathrm{H}$ to synthesize the second strand. Following end repair, a single nucleotide (adenine) was added, and then sequencing adaptors were ligated to the fragments. Finally, the fragments were purified and enriched with PCR amplification to construct cDNA library. Two biological replicates of each sample were used for RNA-Seq via Illumina HiSeq $^{\text {TM }} 2000$ platform by Beijing Genomics Institute (BGI).

\section{Bioinformatics Analysis of RNA-Seq Data}

To acquire clean reads, the low-quality reads, adaptor sequences, and empty reads were removed. All clean reads were mapped with the genome sequences of $T$. salsuginea ${ }^{2}$ using SOAP2 program under the criterion of no more than two mismatches in the alignment ( $\mathrm{Li} \mathrm{R}$. et al., 2009). The gene expression level was calculated using RPKM (Reads per Kb per Million reads)

${ }^{2}$ http://www.phytozome.net/thellungiella.php method according to previous studies (Morrissy et al., 2009; Qu et al., 2016). The relative gene expression level between different samples was calculated by $\log _{2}$ ratio. Differentially expressed genes (DEGs) were identified using NOIseq under the criteria of probability $\geq 0.8$ and the absolute value of $\log ^{2}$ Ratio $\geq 1$. The probability ( $P$-value) was calculated according to the manufacturer's protocol with the default parameters (Tarazona et al., 2011).

Gene Ontology annotation was conducted using Blast2GO (Conesa et al., 2005) by comparing all DEGs with GO terms in the database, which covered three domains: cellular component, molecular function and biological process ${ }^{3}$. The significantly enriched GO terms in DEGs were identified using hypergeometric test comparing to the genome background under the standard of $p$-value $\leq 0.05$. Then GO picture was generated using $\mathrm{WEGO}^{4}$ (Web Gene Ontology Annotation Plot) (Ye et al., 2006). KEGG (Kyoto Encyclopedia of Genes and Genomes) pathway analysis was performed by mapping the DEGs to specific biochemical pathways in KEGG database ${ }^{5}$. Significantly enriched metabolic pathways or signal transduction pathways were identified using enrichment analysis by comparing DEGs with the whole genome background.

\section{qRT-PCR Validation of RNA-Seq Results}

The primers used for qRT-PCR validation were designed using primer premier 5.0 software $^{6}$ and were listed in Supplementary Table S5. The primers were designed according to the transcript

\footnotetext{
${ }^{3}$ http://www.geneontology.org/

${ }^{4}$ http://wego.genomics.org.cn/cgi-bin/wego/index.pl

${ }^{5}$ http://www.genome.jp/kegg/

${ }^{6}$ www.premierbiosoft.com
} 
sequences of $T$. salsuginea downloaded from database ${ }^{7}$. qRTPCR was performed in ABI7500 Real Time System (Applied Biosystems) using SYBR Green I (Roche) with the following reaction: $94^{\circ} \mathrm{C} 10 \mathrm{~min} ; 94^{\circ} \mathrm{C} 15 \mathrm{~s}, 60^{\circ} \mathrm{C} 10 \mathrm{~s}$ and $72^{\circ} \mathrm{C}$ $25 \mathrm{~s}$ for 40 cycles. All reactions were performed in biological triplicates, ubiquitin, and actin were used as internal reference genes. The relative expression of genes was calculated by the software of ABI7500 Real-Time PCR System using the $2^{-\Delta \triangle \mathrm{Ct}}$ method.

\section{AUTHOR CONTRIBUTIONS}

Manuscript draft: JW, CZ, and XW; Analyzing data: CZ and FC; Experiment: JW, QZ, LH, SZ, HX, JQ, TL, and YZ; Conception and supervision of the research: $\mathrm{CZ}$ and XW.

\footnotetext{
${ }^{7}$ https://phytozome.jgi.doe.gov/pz/portal.html我fo?alias=Org_Esalsugineum
}

\section{REFERENCES}

Achard, P., Gong, F., Cheminant, S., Alioua, M., Hedden, P., and Genschik, P. (2008). The cold-inducible CBF1 factor-dependent signaling pathway modulates the accumulation of the growth-repressing DELLA proteins via its effect on gibberellin metabolism. Plant Cell 20, 2117-2129. doi: 10.1105/tpc.108.058941

Achard, P., Vriezen, W. H., Van Der Straeten, D., and Harberd, N. P. (2003) Ethylene regulates Arabidopsis development via the modulation of DELLA protein growth repressor function. Plant Cell 15, 2816-2825. doi: 10.1105/tpc. 015685

Agarwal, M., Hao, Y., Kapoor, A., Dong, C. H., Fujii, H., Zheng, X., et al. (2006). A R2R3 type MYB transcription factor is involved in the cold regulation of CBF genes and in acquired freezing tolerance. J. Biol. Chem. 281, 37636-37645. doi: 10.1074/jbc.M605895200

Agarwal, P. K., Agarwal, P., Reddy, M. K., and Sopory, S. K. (2006). Role of DREB transcription factors in abiotic and biotic stress tolerance in plants. Plant Cell Rep. 25, 1263-1274. doi: 10.1007/s00299-0060204-8

Amtmann, A. (2009). Learning from evolution: Thellungiella generates new knowledge on essential and critical components of abiotic stress tolerance in plants. Mol. Plant 2, 3-12. doi: 10.1093/mp/ ssn094

Boyer, J. S. (1982). Plant productivity and environment. Science 218, 443-448. doi: $10.1126 /$ science.218.4571.443

Chen, W. J., and Zhu, T. (2004). Networks of transcription factors with roles in environmental stress response. Trends Plant Sci. 9, 591-596. doi: 10.1016/j. tplants.2004.10.007

Chinnusamy, V., Ohta, M., Kanrar, S., Lee, B. H., Hong, X., Agarwal, M., et al. (2003). ICE1: a regulator of cold-induced transcriptome and freezing tolerance in Arabidopsis. Genes Dev. 17, 1043-1054. doi: 10.1101/gad. 1077503

Conesa, A., Götz, S., García-Gómez, J. M., Terol, J., Talón, M., and Robles, M. (2005). Blast2go: a universal tool for annotation, visualization and analysis in functional genomics research. Bioinformatics 21, 3674-3676. doi: 10.1093/ bioinformatics/bti610

Dassanayake, M., Oh, D. H., Haas, J. S., Hernandez, A., Hong, H., Ali, S., et al. (2011). The genome of the extremophile crucifer Thellungiella parvula. Nat. Genet. 43, 913-918. doi: 10.1038/ng.889

Elkereamy, A., Bi, Y. M., Ranathunge, K., Beatty, P. H., Good, A. G., and Rothstein, S. J. (2012). The rice R2R3-MYB transcription factor OsMYB55 is involved in the tolerance to high temperature and modulates amino acid metabolism. PLoS ONE 7:e52030. doi: 10.1371/journal.pone.0052030

\section{ACKNOWLEDGMENTS}

This study was supported by the National Natural Science Foundation of China (31500217, 31401034), Natural Science Foundation of Shandong province (ZR2014CQ046, ZR2014CM041), Agricultural Science and Technology Innovation Project (CXGC2016C08) and Young Talents Training Program of Shandong Academy of Agricultural Sciences.

\section{SUPPLEMENTARY MATERIAL}

The Supplementary Material for this article can be found online at: http://journal.frontiersin.org/article/10.3389/fpls.2017.00713/ full\#supplementary-material

FIGURE S1 | Sequence alignment of three COR15 genes.

FIGURE S2 | Hierarchical cluster and correlation analysis between qRT-PCR and RNA-seq.

Eulgem, T., Rushton, P. J., Robatzek, S., and Somssich, I. E. (2000). The WRKY superfamily of plant transcription factors. Trends Plant Sci. 5, 199-206. doi: 10.1016/S1360-1385(00)01600-9

Feng, X. M., Zhao, Q., Zhao, L. L., Qiao, Y., Xie, X. B., Li, H. F., et al. (2012). The cold-induced basic helix-loop-helix transcription factor gene MdCIbHLHlencodes an ICE-like protein in apple. BMC Plant Biol. 12:22. doi: 10.1186/1471-2229-12-22

Gao, F., Zhou, Y., Zhu, W., Li, X., Fan, L., and Zhang, G. (2009). Proteomic analysis of cold stress-responsive proteins in Thellungiella rosette leaves. Planta 230, 1033-1046. doi: 10.1007/s00425-009-1003-6

Gao, M. J., Allard, G., Byass, L., Flanagan, A. M., and Singh, J. (2002). Regulation and characterization of four CBF transcription factors from Brassica napus. Plant Mol. Biol. 49, 459-471. doi: 10.1023/A:1015570308704

Griffith, M., Timonin, M., Wong, A. C. E., Gray, G. R., Akhter, S. R., Saldanha, M., et al. (2007). Thellungiella: an Arabidopsis-related model plant adapted to cold temperatures. Plant Cell Environ. 30, 529-538. doi: 10.1111/j.1365-3040.2007. 01653.x

Guy, C. L. (1990). Cold acclimation and freezing stress tolerance: role of protein metabolism. Annu. Rev. Plant Physiol. 41, 187-223. doi: 10.1146/annurev.pp. 41.060190 .001155

Hajela, R. K., Horvath, D. P., Gilmour, S. J., and Thomashow, M. F. (1990). Molecular cloning and expression of cor (cold-regulated) genes in Arabidopsis thaliana. Plant Physiol. 93, 1246-1252. doi: 10.1104/pp.93.3.1246

Hanano, S., Stracke, R., Jakoby, M., Merkle, T., Domagalska, M. A., Weisshaar, B., et al. (2008). A systematic survey in Arabidopsis thaliana of transcription factors that modulate circadian parameters. BMC Genomics 9:182. doi: 10.1186/14712164-9-182

Harmer, S. L. (2009). The circadian system in higher plants. Annu. Rev. Plant Biol. 60, 357-377. doi: 10.1146/annurev.arplant.043008.092054

Hayat, S., Hayat, Q., Alyemeni, M. N., Wani, A. S., Pichtel, J., and Ahmad, A. (2012). Role of proline under changing environments: a review. Plant Signal. Behav. 7, 1456-1466. doi: 10.4161/psb.21949

Jaglo-Ottosen, K. R., Gilmour, S. J., Zarka, D. G., Schabenberger, O., and Thomashow, M. F. (1998). Arabidopsis CBF1 overexpression induces COR genes and enhances freezing tolerance. Science 280, 104-106. doi: 10.1126/ science.280.5360.104

Kant, S., Kant, P., Raveh, E., and Barak, S. (2006). Evidence that differential gene expression between the halophyte, Thellungiella halophila, and Arabidopsis thaliana is responsible for higher levels of the compatible osmolyte proline and tight control of $\mathrm{Na}+$ uptake in T. halophila. Plant Cell Environ. 29, 1220-1234. doi: 10.1111/j.1365-3040.2006.01502.x

Kawasaki, S., and Bohnert, H. J. (2001). Gene expression profiles during the initial phase of salt stress in rice. Plant Cell 13, 889-905. doi: 10.1105/tpc.13.4.889 
Kim, J. C., Lee, S. H., Cheong, Y. H., Yoo, C. M., Lee, S. I., Chun, H. J., et al. (2001). A novel cold-inducible zinc finger protein from soybean, SCOF-1, enhances cold tolerance in transgenic plants. Plant J. Cell Mol. Biol. 25, 247-259. doi: 10.1046/j.1365-313x.2001.00947.x

Komatsu, S., Yang, G., Khan, M., Onodera, H., Toki, S., and Yamaguchi, M. (2007). Over-expression of calcium-dependent protein kinase 13 and calreticulin interacting protein 1 confers cold tolerance on rice plants. Mol. Genet. Genomics 277, 713-723. doi: 10.1007/s00438-007-0220-6

Lee, B., Henderson, D. A., and Zhu, J. K. (2005). The Arabidopsis coldresponsive transcriptome and its regulation by ICE1. Plant Cell 17, 3155-3175. doi: 10.1105/tpc.105.035568

Leitão, L., Prista, C., Loureiro-Dias, M. C., Moura, T. F., and Soveral, G. (2014). The grapevine tonoplast aquaporin TIP2;1 is a pressure gated water channel. Biochem. Biophys. Res. Commun. 450, 289-294. doi: 10.1016/j.bbrc.2014.05.121

Levitt, J. (1980). Responses of Plants to Environmental Stresses. Cambridge, MA: Academic Press.

Li, D. D., Tai, F. J., Zhang, Z. T., Li, Y., Zheng, Y., Wu, Y. F., et al. (2009). A cotton gene encodes a tonoplast aquaporin that is involved in cell tolerance to cold stress. Gene 438, 26-32. doi: 10.1016/j.gene.2009.02.023

Li, R., Yu, C., Li, Y., Lam, T. W., Yiu, S. M., Kristiansen, K., et al. (2009). SOAP2: an improved ultrafast tool for short read alignment. Bioinformatics 25, 1966-1967. doi: 10.1093/bioinformatics/btp336

Maurel, C. (2007). Plant aquaporins: novel functions and regulation properties. FEBS Lett. 581, 2227-2236. doi: 10.1016/j.febslet.2007.03.021

Morrissy, A. S., Morin, R. D., Delaney, A., Zeng, T., Mcdonald, H., Jones, S., et al. (2009). Next-generation tag sequencing for cancer gene expression profiling. Genome Res. 19, 211-241. doi: 10.1101/gr.094482.109

Nakamura, J., Yuasa, T., Thi, H. T., Harano, K., Tanaka, S., Iwata, T., et al. (2011). Rice homologs of inducer of CBF expression (OsICE) are involved in cold acclimation. Plant Biotechnol. 28, 303-309. doi: 10.5511/plantbiotechnology.11. $0421 \mathrm{a}$

Nover, L., Bharti, K., Döring, P., Mishra, S. K., Ganguli, A., and Scharf, K. D. (2002). Arabidopsis and the heat stress transcription factor world: how many heat stress transcription factors do we need? Cell Stress Chaperones 6, 177-189.

Nuruzzaman, M., Sharoni, A. M., and Kikuchi, S. (2013). Roles of NAC transcription factors in the regulation of biotic and abiotic stress responses in plants. Front. Microbiol. 4:248. doi: 10.3389/fmicb.2013.00248

Park, S., Lee, C. M., Doherty, C. J., Gilmour, S. J., Kim, Y., and Thomashow, M. F. (2015). Regulation of the Arabidopsis cbf regulon by a complex lowtemperature regulatory network. Plant J. 82, 193-207. doi: 10.1111/tpj.12796

Peng, H. H., Shan, W., Kuang, J. F., Lu, W. J., and Chen, J. Y. (2013). Molecular characterization of cold-responsive basic helix-loop-helix transcription factors MabHLHs that interact with MaICE1 in banana fruit. Planta 238, 937-953. doi: 10.1007/s00425-013-1944-7

Qu, C. P., Xu, Z. R., Hu, Y. B., Lu, Y., Yang, C. J., Sun, G. Y., et al. (2016). RNASEQ reveals transcriptional level changes of poplar roots in different forms of nitrogen treatments. Front. Plant Sci. 7:51. doi: 10.3389/fpls.2016.00051

Shi, Y., An, L., Zhang, M., Huang, C., Zhang, H., and Xu, S. (2008). Regulation of the plasma membrane during exposure to low temperatures in suspensioncultured cells from a cryophyte (Chorispora bungeana). Protoplasma 232, 173-181. doi: 10.1007/s00709-008-0291-1

Singh, K. B., Foley, R. C., and Oñate-Sánchez, L. (2002). Transcription factors in plant defense and stress responses. Curr. Opin. Plant Biol. 5, 430-436. doi: 10.1016/S1369-5266(02)00289-3

Su, C. F., Wang, Y. C., Tsaihung, H., Lu, C. A., Tunghai, T., and Yu, S. M. (2010). A novel MYBS3-dependent pathway confers cold tolerance in rice. Plant Physiol. 153, 145-158. doi: 10.1104/pp.110.153015

Tarazona, S., Garcíaalcalde, F., Dopazo, J., Ferrer, A., and Conesa, A. (2011). Differential expression in RNA-seq: a matter of depth. Genome Res. 21, 2213-2223. doi: 10.1101/gr.124321.111

Thomas, D., Patrick, G., Zuther, E., Bettina, S., Hincha, D. K., and Willmitzer, L. (2012). Differential remodeling of the lipidome during cold acclimation in natural accessions of Arabidopsis thaliana. Plant J. Cell Mol. Biol. 72, 972-982. doi: $10.1111 /$ tpj.12007

Thomashow, M. F. (1998). Role of cold-responsive genes in plant freezing tolerance. Plant Physiol. 118, 1-8. doi: 10.1104/pp.118.1.1

Thomashow, M. F. (1999). PLANT COLD ACCLIMATION: freezing tolerance genes and regulatory mechanisms. Annu. Rev. Plant Biol. 50, 571-599. doi: 10.1146/annurev.arplant.50.1.571

Toledoortiz, G., Huq, E., and Quail, P. H. (2003). The Arabidopsis basic/helixloop-helix transcription factor family. Plant Cell 15, 1749-1770. doi: 10.1105/ tpc.013839

Vannini, C., Locatelli, F., Bracale, M., Magnani, E., Marsoni, M., Osnato, M., et al. (2004). Overexpression of the rice Osmyb4 gene increases chilling and freezing tolerance of Arabidopsis thaliana plants. Plant J. 37, 115-127. doi: 10.1046/j. 1365-313X.2003.01938.x

Wanner, L. A., and Junttila, O. (1999). Cold-induced freezing tolerance in Arabidopsis. Plant Physiol. 120, 391-400. doi: 10.1104/pp.120.2.391

Willige, B. C., Ghosh, S., Nill, C., Zourelidou, M., Dohmann, E. M., Maier, A., et al. (2007). The DELLA domain of GA INSENSITIVE mediates the interaction with the GA INSENSITIVE DWARF1A gibberellin receptor of Arabidopsis. Plant Cell 19, 1209-1220. doi: 10.1105/tpc.107.051441

Wisniewski, M., Norelli, J., Bassett, C., Artlip, T., and Macarisin, D. (2011). Ectopic expression of a novel peach (Prunus persica) CBF transcription factor in apple (Malus $\times$ domestica) results in short-day induced dormancy and increased cold hardiness. Planta 233, 971-983. doi: 10.1007/s00425-011-1358-3

Wong, C. E., Li, Y., Labbe, A., Guevara, D., Nuin, P., Whitty, B., et al. (2006). Transcriptional profiling implicates novel interactions between abiotic stress and hormonal responses in Thellungiella, a close relative of Arabidopsis. Plant Physiol. 140, 1437-1450. doi: 10.1104/pp.105.070508

Wu, H. J., Zhang, Z., Wang, J. Y., Oh, D. H., Dassanayake, M., Liu, B., et al. (2012). Insights into salt tolerance from the genome of Thellungiella salsuginea. Proc. Natl. Acad. Sci. U.S.A. 109, 12219-12224. doi: 10.1073/pnas.12099 54109

Xiong, L., Schumaker, K. S., and Zhu, J. K. (2002). Cell signaling during cold, drought, and salt stress. Plant Cell 14, S165-S183.

Xiong, Y., and Fei, S. Z. (2006). Functional and phylogenetic analysis of a DREB/CBF-like gene in perennial ryegrass (Lolium perenne L.). Planta 224, 878-888. doi: 10.1007/s00425-006-0273-5

Xu, W., Zhang, N., Jiao, Y., Li, R., Xiao, D., and Wang, Z. (2014). The grapevine basic helix-loop-helix $(b H L H)$ transcription factor positively modulates CBFpathway and confers tolerance to cold-stress in Arabidopsis. Mol. Biol. Rep. 41, 5329-5342. doi: 10.1007/s11033-014-3404-2

Yamaguchi-Shinozaki, K., and Shinozaki, K. (2001). Improving plant drought, salt and freezing tolerance by gene transfer of a single stress-inducible transcription factor. Novartis Found. Symp. 236, 176-186.

Ye, J., Fang, L., Zheng, H., Zhang, Y., Chen, J., Zhang, Z., et al. (2006). WEGO: a web tool for plotting GO annotations. Nucleic Acids Res. 34, 293-297. doi: 10.1093/nar/gkl031

Zhao, C., Zhang, Z., Xie, S., Si, T., Li, Y., and Zhu, J. K. (2016). Mutational evidence for the critical role of cbf transcription factors in cold acclimation in Arabidopsis. Plant Physiol. 171, 2744-2759. doi: 10.1104/pp.16.00533

Conflict of Interest Statement: The authors declare that the research was conducted in the absence of any commercial or financial relationships that could be construed as a potential conflict of interest.

Copyright (c) 2017 Wang, Zhang, Cui, Hou, Zhao, Xia, Qiu, Li, Zhang, Wang and Zhao. This is an open-access article distributed under the terms of the Creative Commons Attribution License (CC BY). The use, distribution or reproduction in other forums is permitted, provided the original author(s) or licensor are credited and that the original publication in this journal is cited, in accordance with accepted academic practice. No use, distribution or reproduction is permitted which does not comply with these terms. 